\title{
Inhibition of HIV-1 replication by RNA with a microRNA-like function
}

\author{
KEIJIRO KATO $^{1}$, TAKASHI SENOKI ${ }^{1}$ and HIROSHI TAKAKU ${ }^{1,2}$ \\ ${ }^{1}$ Department of Life and Environmental Science and ${ }^{2}$ High Technology Research Center, \\ Chiba Institute of Technology, Narashino, Chiba 275-0016, Japan
}

Received August 10, 2012; Accepted October 11, 2012

DOI: $10.3892 / \mathrm{ijmm} .2012 .1170$

\begin{abstract}
Human immunodeficiency virus type 1 (HIV-1) replication is suppressed by a small guide RNA ( $\operatorname{sgRNA}$ ) that targets the packaging signal of HIV-1 RNA. We unintentionally produced a plasmid with the reverse sequence of the sgRNA and its terminator (pR- $\Psi$-sgRNA-ter). Both sgRNA and R- $\Psi$-sgRNA suppress HIV-1, but the mechanism by which R- $\Psi$-sgRNA suppresses HIV is not clear. To evaluate whether the suppressive effect is caused by an RNA interference or microRNA (miRNA)-like mechanism, R- $\Psi$-sgRNA was synthesized in vitro and treated with the Dicer enzyme, an important enzyme for RNA interference and miRNA. The RNA was cleaved into fragments of approximately 24 nucleotides (nt). We analyzed the sequence of the RNA fragments and predicted the RNA secondary structure of R- $\Psi$-sgRNA to determine the region recognized by the Dicer enzyme. The lengths of the R- $\Psi$-sgRNA fragments ranged from 48 to $140 \mathrm{nt}$, and were predicted to form double strands, including mismatches, in this region. An HIV-1 p24 assay indicated that the R- $\Psi$-sgRNA fragments suppressed HIV-1 replication. These findings suggest that R- $\Psi$-sgRNA acts as a miRNA to inhibit HIV-1.
\end{abstract}

\section{Introduction}

RNA interference (RNAi) refers to the sequence-specific degradation of RNA that follows the cellular introduction of homologous, short interfering RNA (siRNA) (1-4). RNAi

Correspondence to: Professor Hiroshi Takaku, Department of Life and Environmental Science, 2-17-1 Tsudanuma, Narashino, Chiba 275-0016, Japan

E-mail: hiroshi.takaku@it-chiba.ac.jp

Abbreviations: HIV-1, human immunodeficiency virus type 1; miRNA, microRNA; nt, nucleotide; RISC, RNA-induced silencing complex; RNAi, RNA interference; sgRNA, small guide RNA; siRNA, short interfering RNA; tRNase ZL, tRNA 3'-processing endoribonuclease; $\Psi, \mathrm{HIV}-1$ packaging signal; tRNA $^{\text {met }}$, methionine tRNA; Dicer, RNase III-family enzyme

Key words: Dicer, human immunodeficiency virus type 1, microRNA, small-guide RNA, RNA interference, anti-HIV-1 activity has emerged as a powerful tool for probing the function of genes of a known sequence both in vitro and in vivo. Advances in vector design permit the effective expression of siRNA in human cells by transferring short hairpin RNA (shRNA) expression cassettes. Recent studies reported the ability of RNAi to decrease replication of human immunodeficiency virus type 1 (HIV-1) in lymphocytes using siRNA targeting viral (such as tat, gag, rev, env, nef) (5-11) and host (such as CCR5, CD4) (12-14) proteins. Thus, RNAi might potentially be used as a form of genetic therapy for HIV-1 and associated infections. Another type of short RNA, microRNA (miRNA), has also been reported (15-17). miRNAs are transcribed from the genome as the primary miRNA and digested by Drosha RNase III enzyme to produce the precursor-miRNA in the nucleus. The precursor miRNA is then transported to the cytoplasm and digested by Dicer to become mature miRNA. The miRNA is incorporated into RISC and binds to the target RNA, similar to siRNA (18-22). Since the miRNA has mismatches with the target sequence, translation of the target RNA is suppressed. A large number of miRNAs was recently identified in humans. It is estimated that tens of thousands of miRNAs are coded in the genome, and that these miRNAs control protein expression $(18,23)$. miRNAs are important for maintaining cell functions. New short-strand RNAs have also been found (24-33). For example, a piwi-interacting RNA binding a piwi subfamily protein is thought to be involved in silencing retrotransposons in fetal male germ cells via DNA methylation of their regulatory regions (34), whereas endogenous siRNAs from naturally occurring double-stranded RNAs are thought to regulate protein-coding transcripts and retrotransposons in mammalian oocytes (35). Most small RNAs are coded in the non-coding region of the genome, and the more complex the organism, the larger the non-coding region. Further investigation of the functions of the small RNAs is essential for a better understanding of biological systems.

HIV-1 is the most common pathogenic AIDS virus. HIV-1 infects the immune cells by integrating the viral genome into the host cell genome. Viral proteins are expressed by the integrated genome through the host cell genetic expression mechanisms. Viral particles that bud on the cell membrane are then released from the cells. A packaging signal $(\Psi)$ on HIV-1 mRNA is required for incorporation of the HIV-1 RNA genome into a viral particle when the HIV-1 virus forms on a host cell membrane. 
To suppress HIV-1 replication, we constructed a smallguide RNA (sgRNA) expression vector that targets the SL3 of $\Psi$ and evaluated its effect on HIV-1 replication (36-38). The sgRNA is approximately 30 -nucleotides (nt) long and forms a tRNA-like structure with the target RNA in the nucleus, where it cleaves the target RNA by activating the tRNA processing enzyme tRNAZL (38-42).

A plasmid vector with the reverse sequence of the sgRNA and terminator was unintentionally constructed. This reversesequence sgRNA expression vector, $\mathrm{pR}-\Psi$-sgRNA-ter, also inhibited HIV-1 replication (Fig. 1) (43). The R- $\Psi$-sgRNA expressed by the pR- $\Psi$-sgRNA-ter vector is $200 \mathrm{nt}$ long, and, to suppress HIV-1 replication, must contain the reverse sequence of the sgRNA ( $\operatorname{sgRNA-R}$ ), terminator (ter-R), and also the plasmid sequence. The HIV-1 suppressing mechanism of R- $\Psi$-sgRNA, however, remains unclear. Thus, to elucidate the mechanism of HIV-1 suppression by R- $\Psi$-sgRNA, we evaluated the potential involvement of RNAi or miRNA.

\section{Materials and methods}

Cell culture and transfections. HeLa CD4 ${ }^{+}$cells were grown in RPMI-1640 medium (Sigma, St. Louis, MO, USA) supplemented with $10 \%(\mathrm{v} / \mathrm{v})$ heat-inactivated fetal bovine serum (FBS), $50 \mathrm{U} / \mathrm{ml}$ penicillin, and $50 \mathrm{U} / \mathrm{ml}$ streptomycin at $37^{\circ} \mathrm{C}$ in a $5 \% \mathrm{CO}_{2}$ atmosphere. Transfection was carried out using the FuGENE ${ }^{\mathrm{TM}} 6$ reagent (Roche Diagnostics, Indianapolis, IN, USA) or DMRIE-C (Invitrogen, Carlsbad, CA, USA) according to the manufacturer's protocol.

Plasmid construction. To construct pR- $\Psi$-sgRNA-ter, an oligonucleotide consisting of sgRNA and terminator sequences was ligated into the KpnI sites of pL6 vector $(38,44)$ of pSV2neo (L6) (38) with the tRNA ${ }_{i}^{\text {Met }}$ promoter (45). One side of the oligonucleotide was designed not to be digested with KpnI when the oligonucleotide was ligated to KpnI sites of pL6 vector. pR-GagsgRNA-ter was constructed in the same way as pR- $\Psi$-sgRNA-ter. A PCR product to construct pR- $\Psi$-sgRNA-ter-1 was amplified with specific primers (forward, 5'-GGAATTCCAATTCGC AACCTGTGGTAGC-3' and reverse, 5'-GAAGATCTTCC CCCAAAAAAATAAAGCATTTTTTTCACT-3') to pR- $\Psi$ sgRNA-ter. The forward primer had a KpnI restriction site. The reverse primer had a $B g l \mathrm{II}$ restriction site. The PCR product was ligated to the KpnI and BamHI sites of the pL6 vector. pL6-ter served as a negative control.

Luciferase assay. The pNL4-3luc $\Delta$ env (pNL-luc) was an HIV-1 NL4-3-based vector containing a firefly luciferase gene as a reporter (46). The nef gene along with some of the envelope gene sequences of the HIV-1 NL4-3 genome were deleted, and the firefly luciferase gene was inserted. pNL-luc $(0.1 \mu \mathrm{g})$ and either pL6 plasmid pR- $\Psi$-sgRNA-ter, pR-Gag-sgRNAter, pR- $\Psi$-sgRNA-ter-1, pter-R+sgRNA-R+ter, psgRNAR, pter-R or pL6-ter serving as a negative control $(0.1 \mu \mathrm{g})$ were co-transfected with the transfection reagent, FuGENE 6, into HeLa CD4 ${ }^{+}$cells. A PicaGene kit (Toyo Ink Co., Ltd., Tokyo, Japan) and a Luciferase Assay System (Promega, Madison, WI, USA) were used for the luciferase assay. Two days after transfection, the transfected culture cells were treated with cell lysis buffer. Cell lysate was recovered into $1.5-\mathrm{ml}$ tubes to remove the unused components by centrifugation. Lysate was reacted with luminous substrate and the level of fluorescence was measured on a luminometer (Lumat LB 9507; Berthold Technologies, Bad Wildbad, Germany). The amount of firefly luciferase activity was normalized with reference to the protein concentration of the cell lysate. The protein assay was quantitated using the BCA Protein Assay Reagent kit (Thermo Scientific Inc., Rockford, IL, USA), which is based on bicinchoninic acid (BCA).

RNA synthesis and digestion. The DNA template used to synthesize R- $\Psi$-sgRNA was amplified with specific primers (forward, 5'-GACTCGTAATACGACTCACTATAGGCAG AACAGCAGAGTGGCG-3' and reverse, 5'-AATAAAGCAT TTTTTTCACTGCATTCTAGT-3') to the pR- $\Psi$-sgRNA-ter by PCR. The forward primer had a $\mathrm{T} 7$ promoter sequence to transcribe the R- $\Psi$-sgRNA. The amplified DNA template was incubated with T7 RNA polymerase (Invitrogen) and nucleotide triphosphates for $3 \mathrm{~h}$ at $37^{\circ} \mathrm{C}$ and the synthesized

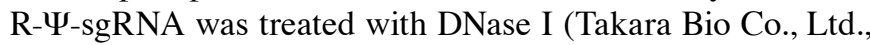
Shiga, Japan) to remove the DNA template. R- $\Psi$-sgRNA was confirmed by $2.0 \%$ denatured formaldehyde agarose gel electrophoresis. R- $\Psi$-sgRNA was incubated with recombinant human Dicer enzyme of BLOCK-iT ${ }^{\mathrm{TM}}$ Dicer RNAi kits (Invitrogen) and purified according to the manufacturer's protocol. Digested RNA fragments were confirmed by $15 \%$ polyacrylamide gel electrophoresis.

Northern blot analysis. Small RNA (<200 nt) was extracted from pR- $\Psi$-sgRNA-ter and siRNA-Dicer $(0,10,20,30 \mathrm{nM})$ co-transfected HeLa CD4 ${ }^{+}$cells using a mirVana ${ }^{\mathrm{TM}}$ miRNA Isolation kit, according to the manufacturer's instructions (Ambion, Foster City, CA, USA). siRNA corresponding to Dicer [5'-UGCUUGAAGCAGCUCUGGA(dTdT)-3'] was purchased from Invitrogen. Small RNA $5 \mu \mathrm{g}$ samples were loaded onto a $15 \%(w / v)$ polyacrylamide/7 $\mathrm{M}$ urea gel. After transfer to a Hybond-N ${ }^{\text {TM }}$ nylon membrane (GE Healthcare Bio-Sciences Corp., Piscataway, NJ, USA), synthetic LNA/DNA oligonucleotides (5'-biotin-TGGATCCCCGGGTACGTCTCC-3') complementary to the antisense strand of the R- $\Psi$-sgRNA region (54-75 nt). The membranes were prehybridized for $1 \mathrm{~h}$ in North2South hybridization buffer (Pierce) at $55^{\circ} \mathrm{C}$ and hybridized overnight to the 5'-biotin labeled LNA/DNA probe (30 $\mathrm{ng} / \mathrm{ml}$ of hybridization buffer). Four posthybridization washes were carried out at $20 \mathrm{~min}$ each at $65^{\circ} \mathrm{C}$ with $2 \mathrm{X} \mathrm{SSC}$, $1 \mathrm{X} \mathrm{SSC}$ is $0.15 \mathrm{M} \mathrm{NaCl}$ plus $0.015 \mathrm{M}$ sodium citrate- $0.1 \%$ sodium dodecyl sulfate (SDS). Detection of LNA/DNA/RNA hybrids was carried out using the North2South chemiluminescent detection system (Thermo Scientific Inc.)

RNA sequence analysis. A poly(A) tail was added to the $3^{\prime}$ terminal ends of the R- $\Psi$-sgRNA fragments with poly(A) polymerase (Ambion, Austin TX, USA) and subsequently ligated to a 5' adapter (5'-ACGGAATTCCTCACTaaa-3'; uppercase letters represent DNA, the lowercase letters represent RNA) to the 5' terminals with T4 RNA ligase (New England Biolabs, Ipswich, MA, USA). Reverse transcription was performed with the poly(A)-specific reverse transcription (RT) primer consisting of a continuous $\mathrm{T}$ sequence and an additional sequence to convert RNA to 


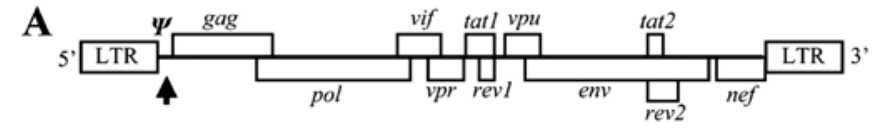

B
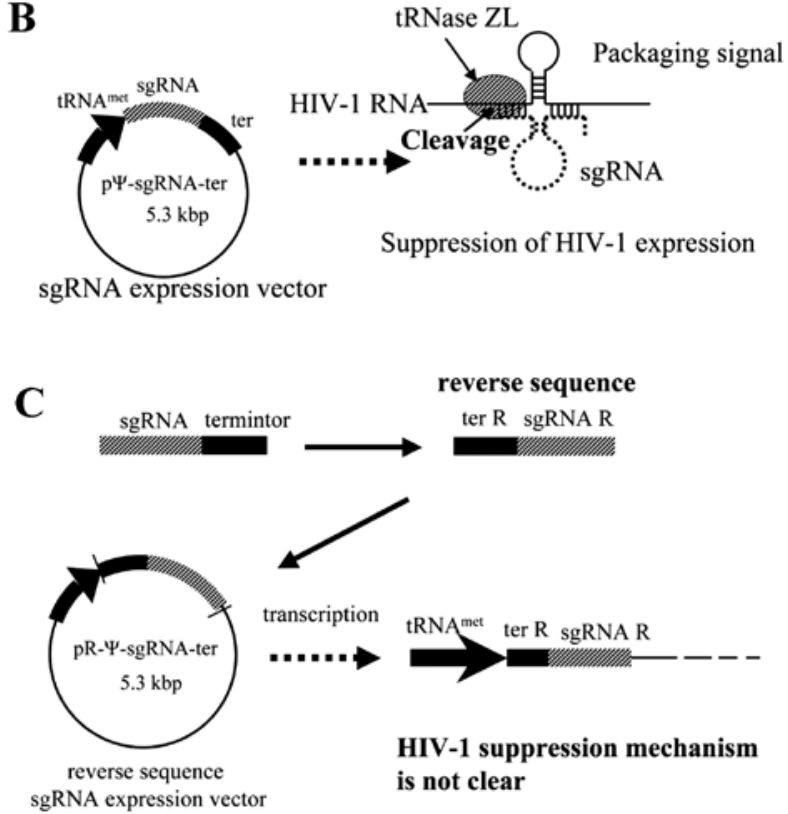

Figure 1. HIV-1 suppression by an sgRNA expression vector and a reverse sequence sgRNA expression vector. (A) Schematic representation of the HIV-1 proviral genome. The HIV-1 packaging signal is indicated by the arrow. (B) HIV-1 suppression by sgRNA/RNase ZL targeting the HIV-1 packaging signal. The packaging signal is important for the incorporation of HIV-1 mRNA into a viral particle. p广-sgRNA-ter expresses the sgRNA that targets the packaging signal and inhibits HIV-1 replication. (C) pR- $\Psi$ sgRNA-ter introduced by the reverse sequence of the insert (ter-R and sgRNA-R) inhibits HIV-1 replication. This plasmid expresses the reverse sequence of the sgRNA, a terminator, and a plasmid (pSV2/neo introduced tRNA $^{\text {met }}$ promoter) sequence.

DNA. The RT primer was as follows: 5'-CTGATCTAGAGGT ACCGGATCCTTTTTTTTTTTTTTTTTTTT-3'. ReverTra- $\alpha$ (Toyobo Co., Ltd., Osaka, Japan) was used for this reaction. The additional sequence of the RT primer was used to adjust the annealing temperature of the following PCR. The reverse transcripts were amplified with $P C R$ primers specific to the 5 ' adapter sequence (5'-CAGCCAACGGAATTCCTCACTAAA-3') and the RT primer by PCR. The PCR products were inserted into the cloning vector, pGEM-Easy vector (Promega), for sequence analysis. Sequence analysis was performed with an ABI PRISM 310 Genetic Analyzer (PE Applied Biosystems, Tokyo, Japan) according to the manufacturer's protocol.

HIV-1 p24 assay. For the HIV-1 p24 assay, HeLa CD4 ${ }^{+}$cells were plated onto 12-well plates (Iwaki Glass, Tokyo, Japan). After $24 \mathrm{~h}$, the medium was replaced with fresh medium and the cells were transfected with small RNA fragments of R- $\Psi$-sgRNA [ $(0.5,0.25$ and $0.05 \mu \mathrm{g})$ and HIV-1 pNL4-3 $(0.05 \mu \mathrm{g})]$. The transfection agents were DMRIE-C (Invitrogen) and FuGENE 6 (Roche Diagnostics). Two days later, HIV-1 gag p24 antigen levels were measured using a chemiluminescent enzyme immunoassay (Lumipulse; Fujirebio, Tokyo, Japan) (47).

\section{Results and Discussion}

Inhibition of HIV-1 replication with the reverse sequence sgRNA expression vector. Recently, we demonstrated the inhibition of HIV-1 gene products in cultured cells by inducing HIV-1 mRNA cleavage using a modified 5'-half-tRNA ${ }^{\mathrm{Arg}}$ (sgRNA) and mammalian tRNA 3'-processing endoribonuclease (tRNase ZL) (38). The sgRNA/target HIV-1RNA complex formed a pre-tRNA-like structure with 5'-half-tRNA and a stable hairpin (3'-half-tRNA) structure resembling the T stem-loop region (Fig. 1A and B). The tRNA ${ }^{\text {met }}$-sgRNA transcript was expressed at high levels and localized in the nucleus. The greatest inhibitory effect on HIV-1 expression was achieved using sgRNA targeting the HIV-1 packaging $(\Psi)$ signal gene (36-38). To construct the sgRNA expression plasmid, a pL6 vector, dpSV/neo with a human methionine tRNA promoter $(38,45)$, was used to insert the sgRNA and terminator sequences. During this process, $\mathrm{pR}-\Psi$-sgRNA-ter, which has the reverse sequence of the sgRNA and the terminator, was unintentionally constructed (Fig. 1C). A transient-expression assay was used to test the ability of pR- $\Psi$-sgRNA-ter to inhibit HIV-1 expression. HeLa $\mathrm{CD} 4{ }^{+}$cells were co-transfected with the sgRNA plasmids and an HIV-1 $1_{\mathrm{NL} 4-3}$ based-vector containing a luciferase-expression marker (pNL-luc) (Fig. 2A) (46), and then HIV-1 suppression was assessed with a single-cycle infectivity assay. HIV-1 $1_{\mathrm{NL4}-3}$ based-luciferase activity was recorded using the control plasmid vector pL6-ter with only the tRNA ${ }^{\text {met }}$ promoter and terminator sequences, rather than the sgRNA-expression plasmid. R- $\Psi$-sgRNA-ter showed good inhibition of HIV-1 expression in cultured cells (Fig. 2C). The contribution of the R- $\Psi$-sgRNA-ter to the anti-HIV-1 effect was examined by three types of modified R- $\Psi$-sgRNA-ter plasmids (R- $\Psi$-sgRNA-ter that did not contain ter-R+sgRNA-R+ter, sgRNA-R or ter-R) as defective pR- $\Psi$-sgRNA-ters (Fig. 2B). We also constructed a control R-sgRNA, pR-Gag-sgRNAter containing the reverse sequence of the sgRNA targeting the HIV-1 gag gene (gag initiation codon site) (Fig. 2B). The luciferase assay indicated that the modified $\mathrm{R}-\Psi$-sgRNA-ter plasmids without the sgRNA-R, ter-R, or plasmid sequence (terR+sgRNA-R+ter) and the control sgRNA, R-Gag-sgRNA-ter did not suppress HIV-1 expression (Fig. 2C). These results suggest that the R- $\Psi$-sgRNA-ter sequence is critical to HIV-1 specific expression.

Length of $R-\Psi$-sgRNA and its inhibitory effect on HIV-I replication. The $\mathrm{pR}-\Psi$-sgRNA-ter termination signal sequence was also reversed, and $\mathrm{pR}-\Psi$-sgRNA-ter did not have a terminator. Therefore we could not predict the length of the R- $\Psi$-sgRNA transcribed from pR- $\Psi$-sgRNA-ter. To determine the length of the R- $\Psi$-sgRNA transcribed from pR- $\Psi$-sgRNA-ter, an RNA sequence analysis was performed. The total RNA of pR- $\Psi$-sgRNA-ter transfected cells was treated with poly(A) polymerase to add a poly(A) tail to the 3 ' terminal end of R- $\Psi$-sgRNA. A PCR primer specific to the transcribed tRNA ${ }^{\text {met }}$ promoter sequence and RT primer specific to the poly(A) tail were used for RT-PCR. A cloning vector was used to insert the PCR products, and the sequences were analyzed. RNA sequence analysis revealed that the R- $\Psi$-sgRNA-ter was $200 \mathrm{nt}$ long (Fig. 3A and B). We confirmed that R- $\Psi$-sgRNA contained sgRNA-R, ter-R, and 
A

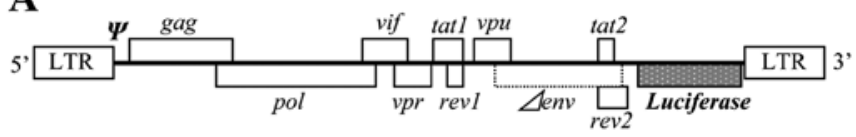

B

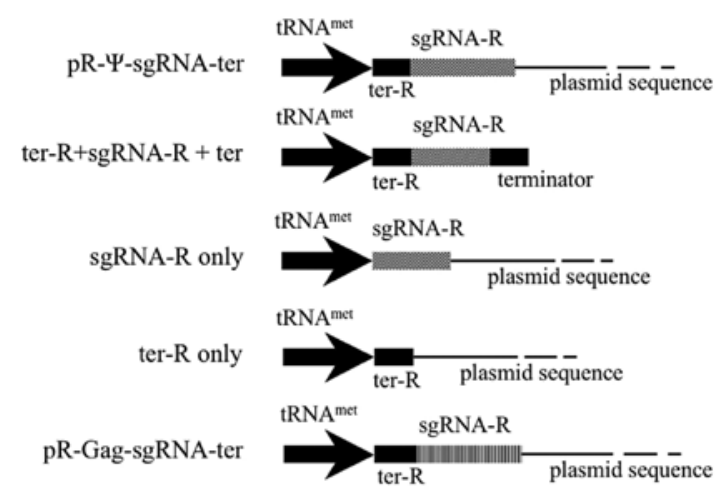

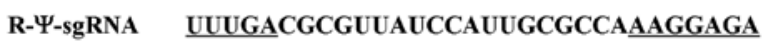

R-Gag-sgRNA AGAUGCGCGUUAUCCAUUGCGCCAGGUAUUA

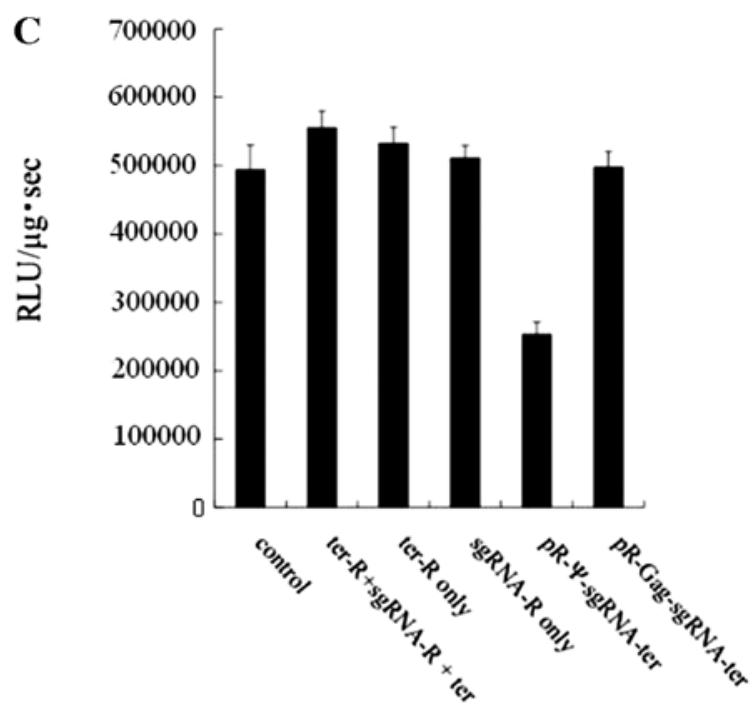

Figure 2. HIV-1 suppression with defective reverse sequence sgRNA expression vectors and reverse sequence sgRNA expression vectors targeting the HIV-1 gag gene. (A) Schematic representation of the pNL-luc. The nef gene and some of the envelope gene sequences in the HIV-1 pNL4-3 genome were deleted, and a firefly luciferase gene was inserted as a reporter. (B) Schematic representation of three defective plasmids and reverse sequence sgRNA expression vectors. Ter-R or sgRNA-R was deleted from $\mathrm{pR}-\Psi$-sgRNA-ter. A terminator was introduced into sgRNA-R downstream of $\mathrm{pR}-\Psi$-sgRNA-ter The sgRNA-R sequence of $\mathrm{pR}-\Psi$-sgRNA-ter and $\mathrm{pR}-\mathrm{Gag}$-sgRNA-ter are indicated at the bottom of (B). (C) Evaluation of HIV-1 suppression with the defective plasmids and pR-Gag-sgRNA-ter by luciferase assay. Plasmids were transfected into $\mathrm{HeLa} \mathrm{CD} 4^{+}$cells. Transfected cells were treated with cell lysis buffer and the level of fluorescence was measured on a luminometer. The results are representative of three independent experiments, and error bars show the standard deviation of the means.

a plasmid sequence following sgRNA-R. A plasmid vector expressing the R- $\Psi$-sgRNA-ter-1 (200 nt) sequence was constructed with pL6 vector, and a luciferase assay indicated that the R- $\Psi$-sgRNA-ter-1 (200 nt) suppressed HIV-1 expression (Fig. 3C). Size-selected RNAs were electrophoresed in
A R- $\Psi$-sgRNA

5'-GGCAGAACAGCAGAGUGGCGCAGCGGAAGCGUGCUGGGCCCAUAA CCCAGAGGUCGAUGGAUCGAAACCGGUACCCCAAAAACGUUUGACGC GUUAUCCAUUGCGCCAAAGGAGACGUACCCGGGGAUCCAGACAUGAU AAGAUACAUUGAUGAGUUUGGACAAACCACAACUAGAAUGCAGUGAAA AAAAUGCUUUAUU-3'

B pR- $\Psi$-sgRNA-ter-1

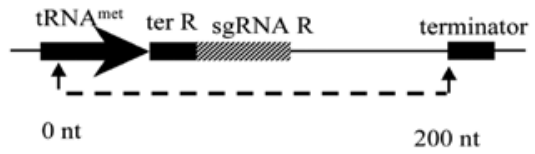

C

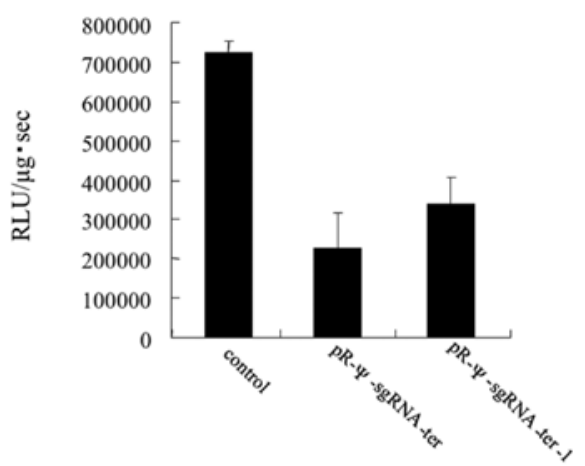

D

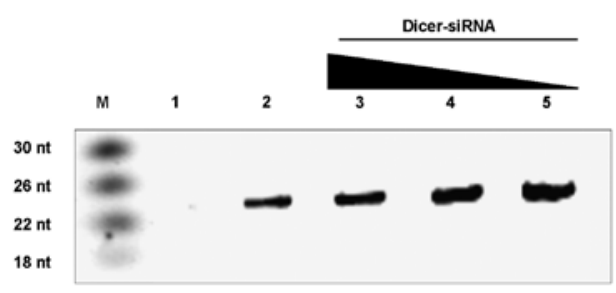

Figure 3. Evaluation of the RNA sequence expressed by $p R-\Psi-s g R N A-t e r$ (A) The RNA sequence expressed by pR- $\Psi$-sgRNA-ter was confirmed by sequence analysis. The RNA sequence was termed R- $\Psi$-sgRNA. (B) The pR- $\Psi$-sgRNA-ter- 1 construct. The terminator was introduced at the 3 terminal of R- $\Psi$-sgRNA. (C) Evaluation of HIV-1 suppression with pR- $\Psi$ sgRNA-ter-1 using a luciferase assay. Plasmids were transfected into $\mathrm{HeLa} \mathrm{CD} 4^{+}$cells. Transfected cells were treated with cell lysis buffer and the level of fluorescence was measured on a luminometer. The results are representative of three independent experiments, and error bars show the standard deviation of the means. (D) HeLa CD4 $4^{+}$cells were transfected with pR- $\Psi$-sgRNA-ter and Dicer-siRNA (10-30 nM). Small RNAs were purified and detected by RNA markers from Ambion (M) or a synthetic LNA/DNA oligonucleotide (5'-biotin-TGGATCCCCGGGTACGTCTCC-3') complementary to the antisense strand of the R- $\Psi$-sgRNA region (54-75 nt) were used to detect a $24 \mathrm{bp}$ signal (lane 2) not found in control ter-R cells (lane 1). Furthermore, HeLa CD4 ${ }^{+}$cells were transfected with pR- $\Psi$-sgRNA-ter and Dicer-siRNA (334-352 nt, 10-30 nM). The expected small RNA decreased in a dose-dependent manner (lanes 3-5).

polyacrylamide gels and transferred to nylon membranes. We then hybridized the membranes with a synthetic LNA/DNA oligonucleotide (5'-biotin-TGGATCCCCGGGTACGTCTCC-3') complementary to the antisense strand of the R- $\Psi$-sgRNA region (54-75 nt) and detected a 24 bp signal (Fig. 3D, lane 2) not seen in the control-ter-R cells (Fig. 3D, lane 1). Furthermore, $\mathrm{HeLa} \mathrm{CD} 4^{+}$cells were transfected with pR- $\Psi$-sgRNA-ter and Dicer-siRNA (334-352 nt, 10-30 nM) and the expected small RNA decreased in a dose-dependent manner (Fig. 3D, lanes 3-5). These results suggest that the 
A

$\begin{array}{lcc}\text { A } & \text { terR } & \text { SgRNA R } \\ \text { (49)-AGAGGUCGAUGGAUCGAAACCGGUUACCCCAAAAACGUUUGACGCG }\end{array}$

UUAUCCAUUGCGCCAAAGGAGACGUACCCGGGGAUCCAGACAUGAUA-(140)

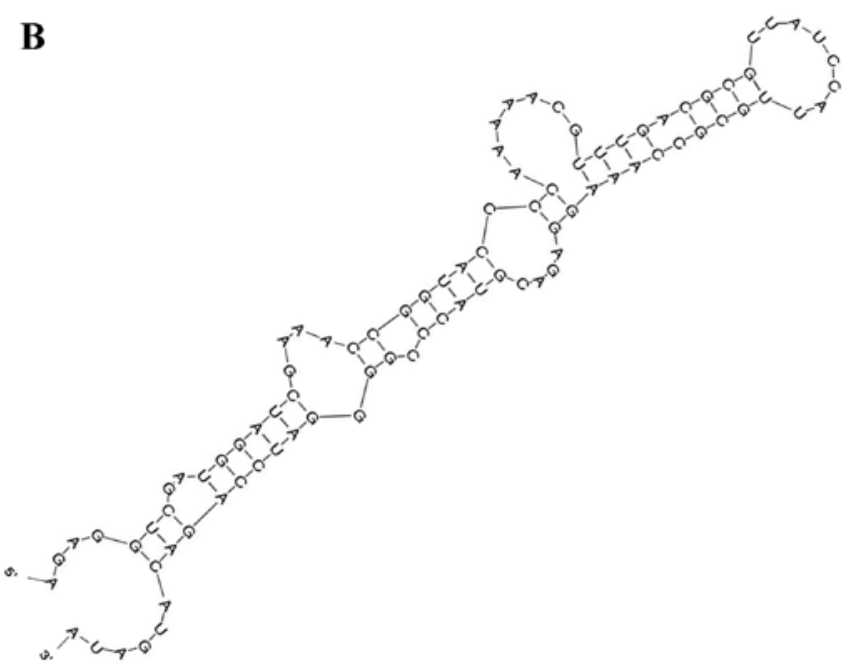

Figure 4. Sequence analysis of diced R- $\Psi$-sgRNA and prediction of the RNA structure recognized by Dicer. (A) The region of R- $\Psi$-sgRNA recognizing by Dicer predicted from sequence analysis of diced R- $\Psi$-sgRNA. Most of the detected sequences were found in nucleotides 49-140 of R- $\Psi$-sgRNA. The functional sequences predicted from the sequence analysis are indicated boldface. (B) The predicted RNA structure recognized by Dicer. The RNA structure of this region was predicted using the RNA Mfold program.

R- $\Psi$-sgRNA-ter-1 (200 nt) sequence is required for HIV-1 suppression.

Sequence analysis of the digested RNA fragments. To search for the double-stranded region recognized by Dicer, an RNA sequence analysis was performed. A poly(A) tail and 5 ' adapter were added to the digested RNA fragments and the RNA was converted to DNA by RT-PCR. A cloning vector was used to insert the DNA fragments and the sequences were analyzed. Most of the RNA fragment sequences existed in two regions of the $\mathrm{R}-\Psi$-sgRNA: the region from ter-R to the anterior sequence of ter-R, which was transcribed initially by the tRNA promoter, and the region from sgRNA-R to the plasmid sequence following sgRNA-R (Fig. 4A). In addition, the sgRNA-R sequences obtained from the sequence analysis were not present in the sgRNA-R region of the pR-GagsgRNA-ter targeting gag gene. These results, therefore, seemed to be related to the use of defective $p R-\Psi$-sgRNA-ter and pR-Gag-sgRNA-ter in the assay (Fig. 2).

The RNA secondary structure of R- $\Psi$-sgRNA was predicted using the Mfold program (48) and the doublestranded region was searched based on the RNA sequence data obtained from the sequence analysis. The double-stranded region was located between 48 to $140 \mathrm{nt}$ of the R- $\Psi$-sgRNA, and the presence of sequences of short-strand RNA fragments in this region was confirmed (Fig. 4B). Furthermore, miRNAs recognize HIV-1 NL4-3 target mRNAs by their hybridization pattern as predicted using the miRNA target scanning
A

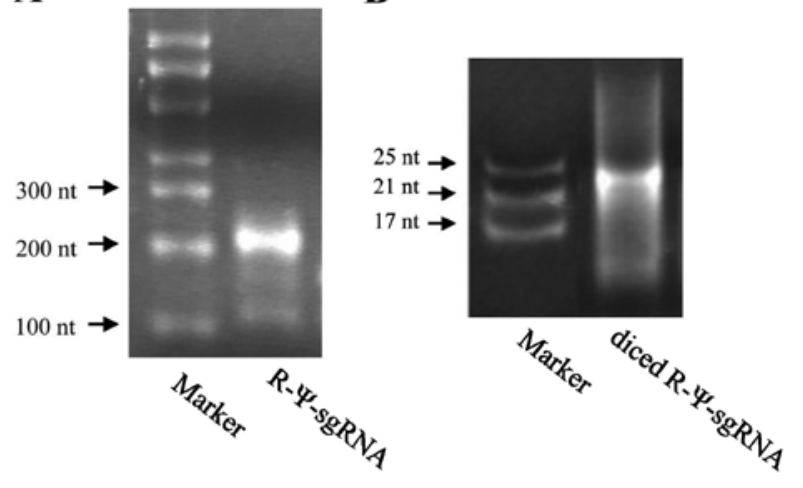

C

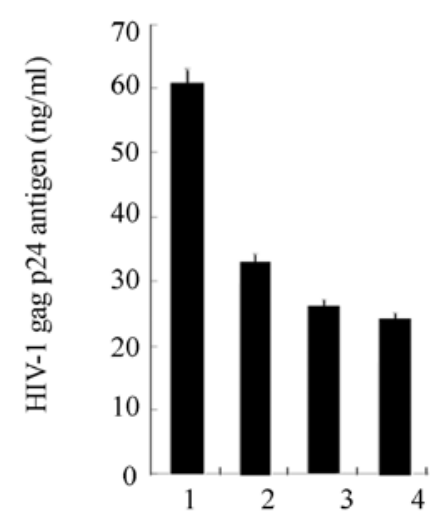

Figure 5. Evaluation of anti-HIV activity with diced R- $\Psi$-sgRNA. (A) Synthesis of R- $\Psi$-sgRNA by T7 RNA polymerase. A DNA template of R- $\Psi$-sgRNA was incubated with T7 RNA polymerase and nucleotide triphosphates for $3 \mathrm{~h}$ at $37^{\circ} \mathrm{C}$. R- $\Psi$-sgRNA was confirmed by $2.0 \%$ denature formaldehyde agarose gel electrophoresis. (B) Digestion of R- $\Psi$-sgRNA by Dicer. R- $\Psi$-sgRNA was digested with the recombinant human Dicer enzyme according to the manufacturer's protocol. Diced R- $\Psi$-sgRNA was confirmed by $15 \%$ polyacrylamide gel electrophoresis. (C) Evaluation of HIV-1 suppression by HIV-1 gag p24 antigen levels. HeLa CD4 ${ }^{+}$cells co-transfected with diced R- $\Psi$-sgRNA and HIV-1 pNLE4-3 were tested for HIV-1 gag p24 antigen expression using an automated ELISA system (47). The results are representative of three independent experiments, and error bars show the standard deviation of the means. Lane 1, pNL4-3 (0.05 $\mu \mathrm{g})$; lane 2, diced R- $\Psi$-sgRNA-pNL4-3 (0.05 $\mu \mathrm{g}: 0.05 \mu \mathrm{g})$; lane 3, diced R- $\Psi$-sgRNA-pNL4-3 (0.25 $\mu \mathrm{g}: 0.05 \mu \mathrm{g})$; lane 4, diced R- $\Psi$ sgRNA-pNL4-3 (0.5 $\mu \mathrm{g}: 0.05 \mu \mathrm{g})$.

algorithm program (49). The miRNA region was searched based on the $24 \mathrm{nt}$ small RNA shown in Fig. 3D, and the presence of miRNA in the double-stranded region (nucleotides 48-140) of R- $\Psi$-sgRN was confirmed (data not shown). The double-stranded region had mismatches, indicating that the short-strand RNA fragments include miRNA, but not siRNA.

Digestion of R- $\Psi$-sgRNA with Dicer. We hypothesized that the secondary structure of the R- $\Psi$-sgRNA had double-stranded regions that may be recognized by the Dicer RNase III enzyme, which produces the 24 nt long RNA fragments that are important for RNAi and miRNA. To examine whether the HIV-1 suppression mechanism of R- $\Psi$-sgRNA involved RNAi or miRNA, R- $\Psi$-sgRNA was synthesized in vitro and digested with Dicer (50). The size of the digested RNA fragments was confirmed with $15 \%$ polyacrylamide gel electrophoresis (Fig. 5A and B). 
Short-strand RNA fragments inhibit HIV-1 replication. To test the inhibitory activity of the short-strand RNA fragments on HIV-1 replication in a transient assay, an HIV-1 expression plasmid (pNL4-3) and the short-strand RNA fragments were transfected into HeLa CD4 ${ }^{+}$cells. The virus production in the culture supernatant was monitored with the HIV-1 p24 antigen (HIV-1 gag gene product) assay. The results of the HIV-1 p24 assay indicated that these RNA fragments had anti-HIV activity and suppressed HIV-1 replication. Furthermore, our analysis revealed that the short-strand RNA fragments dosedependently inhibited HIV-1 replication (Fig. 5C). These results suggest that the short-strand RNA fragments have a miRNA-like effect on the HIV-1 virus.

In conclusion, the short-strand RNA fragments (24 nt long) of R- $\Psi$-sgRNA cleaved using the Dicer enzyme exhibited antiHIV-1 activity. The sequences of these RNA fragments were analyzed to determine the critical sequence for the anti-HIV-1 activity. These findings suggest that R- $\Psi-\operatorname{sgRNA}$ acts as a miRNA to inhibit HIV-1.

\section{Acknowledgements}

The authors thank Dr I.S.Y. Chen for providing pNL4-3luc $\Delta$ env and Makoto Abe and Yuji Yokota for their technical assistance. We also thank Dr Y. Habu for the valuable discussion. This study was supported in part by a Grant-in-Aid for AIDS research from the Ministry of Health, Labor, and Welfare, Japan, by a Grant from the Strategic Research Foundation Grant-aided Project for Private Universities from the Ministry of Education, Culture, Sport, Science and Technology, Japan (MEXT), and by a Grant-in-Aid for Science Research (C) from Japan Society for the Promotion of Science (JSPS), Japan.

\section{References}

1. Fire A, Xu S, Montgomery MK, Kostas SA, Driver SE and Mello CC: Potent and specific genetic interference by doublestranded RNA in Caenorhabditis elegans. Nature 39: 806-811, 1998.

2. Elbashir SM, Harborth J, Lendeckel W, Yalcin A, Weber K and Tuschl T: Duplexes of 21-nucleotide RNAs mediate RNA interference in cultured mammalian cells. Nature 411: 494-498, 2001.

3. Sharp PA: RNA interference. Genes Dev 15: 485-490, 2001.

4. Hannon GJ: RNA interference. Nature 418: 244-251, 2002.

5. Coburn GA and Cullen BR: Potent and specific inhibition of human immunodeficiency virus type 1 replication by RNA interference. J Virol 76: 9225-9231, 2002.

6. Jacque JM, Trioques K and Stevenson M: Modulation of HIV-1 replication by RNA interference. Nature 418: 379-380, 2002.

7. Lee NS, Dohjima T, Bauer G, Li H, Li MJ, Ehsani A, Salvaterra P and Rossi J: Expression of small interfering RNAs targeted against HIV-1 rev transcripts in human cells. Nat Biotech 19: 500-505, 2002.

8. Novina CD, Murray MF, Dykxhoorn DM, Beresford PJ, Riess J, Lee SK, Collman RG, Lieberman J, Shankar P and Sharp PA: siRNA-directed inhibition of HIV-1 infection. Nat Med 8: 681-686, 2002

9. Yamamoto T, Omoto S, Mizuguchi M, Mizukami H, Okuyama H, Okada N, Saksena NK, Brisibe EA, Otake K and Fuji YR: Double-stranded nef RNA interferes with human immunodeficiency virus type 1 replication. Microbiol Immunol 46: 809-817, 2002.

10. Song E, Lee SK, Dykxhoorn DM, Novina C, Zhang D, Crawford K, Cerny J, Sharp PA, Lieberman J, Manjunath N and Shankar P: Sustained small interfering RNA-mediated human immunodeficiency virus type 1 inhibition in primary macrophages. J Virol 77: 7174-7181, 2003.
11. Park WS, Hayafune M, Miyano-Kurosaki N and Takaku H: Specific HIV-1 env gene silencing by small interfering RNAs in human peripheral blood mononuclear cells. Gene Ther 10: 2046-2050, 2003.

12. Martínez MA, Gutiérrez A, Armand-Ugón M, Blanco J, Parera M, Gómez J, Clotet B and Esté JA: Suppression of chemokine receptor expression by RNA interference allows for inhibition of HIV-1 replication. AIDS 16: 2385-2390, 2002.

13. Qin XF, An DS, Chen IS and Baltimore D: Inhibiting HIV-1 infection in human $T$ cells by lentiviral-mediated delivery of small interfering RNA against CCR5. Proc Natl Acad Sci USA 100: 183-188, 2002.

14. Anderson J, Banerjea A and Akkina R: Suppression of HIV-1 infection by a stem-loop structured anti-CXCR4 siRNA. AIDS Res Hum Retroviruses 19: 699-706, 2003.

15. Lee RC, Feinbaum RL and Ambros V: The C. elegans heterochronic gene lin-4 encodes small RNAs with antisense complementarity to lin-14. Cell 75: 843-854, 1993.

16. Lagos-Quintana M, Rauhut R, Lendeckel $\mathrm{W}$ and Tuschl T: Identification of novel genes coding for small expressed RNAs. Science 294: 853-858, 2001.

17. Lau NC, Lim LP, Weinstein EG and Bartel DP: An abundant class of tiny RNAs with probable regulatory roles in Caenorhabditis elegans. Science 294: 858-862, 2001.

18. Bartel DP: MicroRNAs: genomics, biogenesis, mechanism, and function. Cell 116: 281-297, 2004.

19. Hammond SM: Dicing and slicing the core machinery of the RNA interference pathway. FEBS Lett 579: 5822-5829, 2005

20. Tang G: siRNA and miRNA: an insight into RISCs. Trends Biochem Sci 30: 106-114, 2005.

21. Tomari Y and Zamore PD: Perspective: machines for RNAi. Genes Dev 19: 517-529, 2005.

22. Kim VN: MicroRNA biogenesis: coordinated cropping and dicing. Nat Rev Mol Cell Biol 6: 376-385, 2005.

23. Shiomi M: Functions and regulatory mechanisms of small RNAs expressed in living organisms. Exp Med 25: 794-799, 2007.

24. Aravin AA, Lagos-Quintana M, Yalcin A, Zavolan M, Marks D, Snyder B, Gaasterland T, Meyer J and Tuschl T: The small RNA profile during Drosophila melanogaster development. Dev Cell 5: 337-350, 2003.

25. Aravin A, Gaidatzis D, Pfeffer S, Lagos-Quintana M, Landgraf P, Iovino N, Morris P, Brownstein MJ, Kuramochi-Miyagawa S, Nakano T, Chien M, Russo JJ, Ju J, Sheridan R, Sander C, Zavolan M and Tuschl T: A novel class of small RNAs bind to MILI protein in mouse testes. Nature 442: 203-207, 2006.

26. Girard A, Sachidanandam R, Hannon GJ and Carmell MA: A germline-specific class of small RNAs binds mammalian Piwi proteins. Nature 442: 199-202, 2006.

27. Grivna ST, Beyret E, Wang Z and Lin H: A novel class of small RNAs in mouse spermatogenic cells. Genes Dev 20: 1709-1714, 2006.

28. Lau NC, Seto AG, Kim J, Kuramochi-Miyagawa S, Nakano T, Bartel DP and Kingston RE: Characterization of the piRNA complex from rat testes. Science 313: 363-367, 2006.

29. Saito K, Nishida KM, Mori T, Kawamura Y, Miyoshi K, Nagami T, Siomi H and Siomi MC: Specific association of Piwi with rasiRNAs derived from retrotransposon and heterochromatic regions in the Drosophila genome. Genes Dev 20: 2214-2222, 2006

30. Vagin VV, Sigova A, Li C, Seitz H, Gvozdev V and Zamore PD: A distinct small RNA pathway silences selfish genetic elements in the germline. Science 313: 320-324, 2006.

31. Watanabe T, Takeda A, Tsukiyama T, Mise K, Okuno T, Sasaki H, Minami N and Imai H: Identification and characterization of two novel classes of small RNAs in the mouse germline: retrotransposon-derived siRNAs in oocytes and germline small RNAs in testes. Genes Dev 20: 1732-1743, 2006.

32. Nishida KM, Saito K, Mori T, Kawamura Y, Nagami-Okada T, Inagaki S, Siomi H and Siomi MC: Gene silencing mechanisms mediated by Aubergine piRNA complexes in Drosophila male gonad. RNA 13: 1911-1922, 2007.

33. Klattenhoff $\mathrm{C}$ and Theurkauf $\mathrm{W}$ : Biogenesis and germline functions of piRNAs. Development 135: 3-9, 2008

34. Kuramochi-Miyagawa S, Watanabe T, Gotoh K, Totoki Y, Toyoda A, Ikawa M, Asada N, Kojima K, Yamaguchi Y, Ijiri TW, Hata K, Li E, Matsuda Y, Kimura T, Okabe M, Sakaki Y, Sasaki $\mathrm{H}$ and Nakano T: DNA methylation of retrotransposon genes is regulated by Piwi family members MILI and MIWI2 in murine fetal testes. Genes Dev 22: 908-917, 2008. 
35. Watanabe T, Totoki Y, Toyoda A, Kaneda M, KuramochiMiyagawa S, Obata Y, Chiba H, Kohara Y, Kono T, Nakano T, Surani MA, Sakaki Y and Sasaki H: Endogenous siRNAs from naturally formed dsRNAs regulate transcripts in mouse oocytes. Nature 453: 539-543, 2008.

36. Clever JL, Miranda D Jr and Parslow TG: RNA structure and packaging signals in the 5 leader region of the human immunodeficiency virus type 1 genome. J Virol 76: 12381-12387, 2002.

37. Russell RS, Hu J, Beriault V, Mouland AJ, Laughrea M, Kleiman L, Wainberg MA and Liang C: Sequences downstream of the 5 splice donor site are required for both packaging and dimerization of human immunodeficiency virus type 1 RNA. J Virol 77: 84-96, 2003.

38. Habu Y, Miyano-Kurosaki N, Kitano M, Endo Y, Yukita M, Ohira S, Takaku H, Nashimoto $\mathrm{M}$ and Takaku H: Inhibition of HIV-1 gene expression by retroviral vector-mediated small-guide RNAs that direct specific RNA cleavage by tRNase ZL. Nucleic Acids Res 33: 235-243, 2005.

39. Nashimoto M: Conversion of mammalian tRNA 3' processing endoribonuclease to four-base-recognizing RNA cutters. Nucleic Acids Res 23: 3642-3647, 1995.

40. Nashimoto M: Specific cleavage of target RNAs from HIV-1 with $5^{\prime}$ half tRNA by mammalian tRNA 3 ' processing endoribonuclease. RNA 2: 523-524, 1996.

41. Takaku H, Minagawa A, Takagi $M$ and Nashimoto $M$ : A candidate prostate cancer susceptibility gene encodes tRNA 3 processing endoribonuclease. Nucleic Acids Res 31: 2272-2278, 2003.

42. Takaku H, Minagawa A, Takagi $M$ and Nashimoto $M$ : The $\mathrm{N}$-terminal half-domain of the long form of tRNase $\mathrm{Z}$ is required for the RNase 65 activity. Nucleic Acids Res 32: 4429-4438, 2004.
43. Kato K, Habu Y and Takaku H: Micro RNA like inhibition of HIV-1 replication. Nucleic Acids Symp Ser (Oxf) 52: 509-510, 2008.

44. Sugiyama R, Hayafune M, Habu Y, Yamamoto N and Takaku H: HIV-1 RT-dependent DNAzyme expression inhibits HIV-1 replication without the emergence of escape viruses. Nucleic Acids Res 39: 589-598, 2011.

45. Keith G, Heitzler J, el Adlouni C, Glasser AL, Fix C, Desgres J and Dirheimer G: The primary structure of cytoplasmic initiator tRNA(Met) from Schizosaccharomyces pombe. Nucleic Acids Res 21: 2949, 1993.

46. Planelles V, Bachelerie F, Jowett JB, Haislip A, Xie Y, Banooni P, Masuda T and Chen IS: Fate of the human immunodeficiency virus type 1 provirus in infected cells: a role for vpr. J Virol 69: 5883-5889, 1995.

47. Sakai A, Hirabayashi Y, Aizawa S, Tanaka M, Ida S and Oka S: Investigation of a new p24 antigen detection system by the chemiluminescence-enzyme-immuno-assay. Kansenshogaku Zasshi 73: 205-212, 1999 (In Japanese).

48. Zuker M: Mfold web server for nucleic acid folding and hybridization prediction. Nucleic Acids Res 31: 3406-3415, 2003.

49. Enright AJ, John B, Tushl T, Sander C and Marks DS: Micro RNA targets in Drosophila. Genom Biol 5: R1, 2003.

50. Myers JW, Jones JT, Meyer T and Ferrell JE Jr: Recombinant Dicer efficiently converts large dsRNAs into siRNAs suitable for gene silencing. Nat Biotechnol 3: 324-328, 2003. 